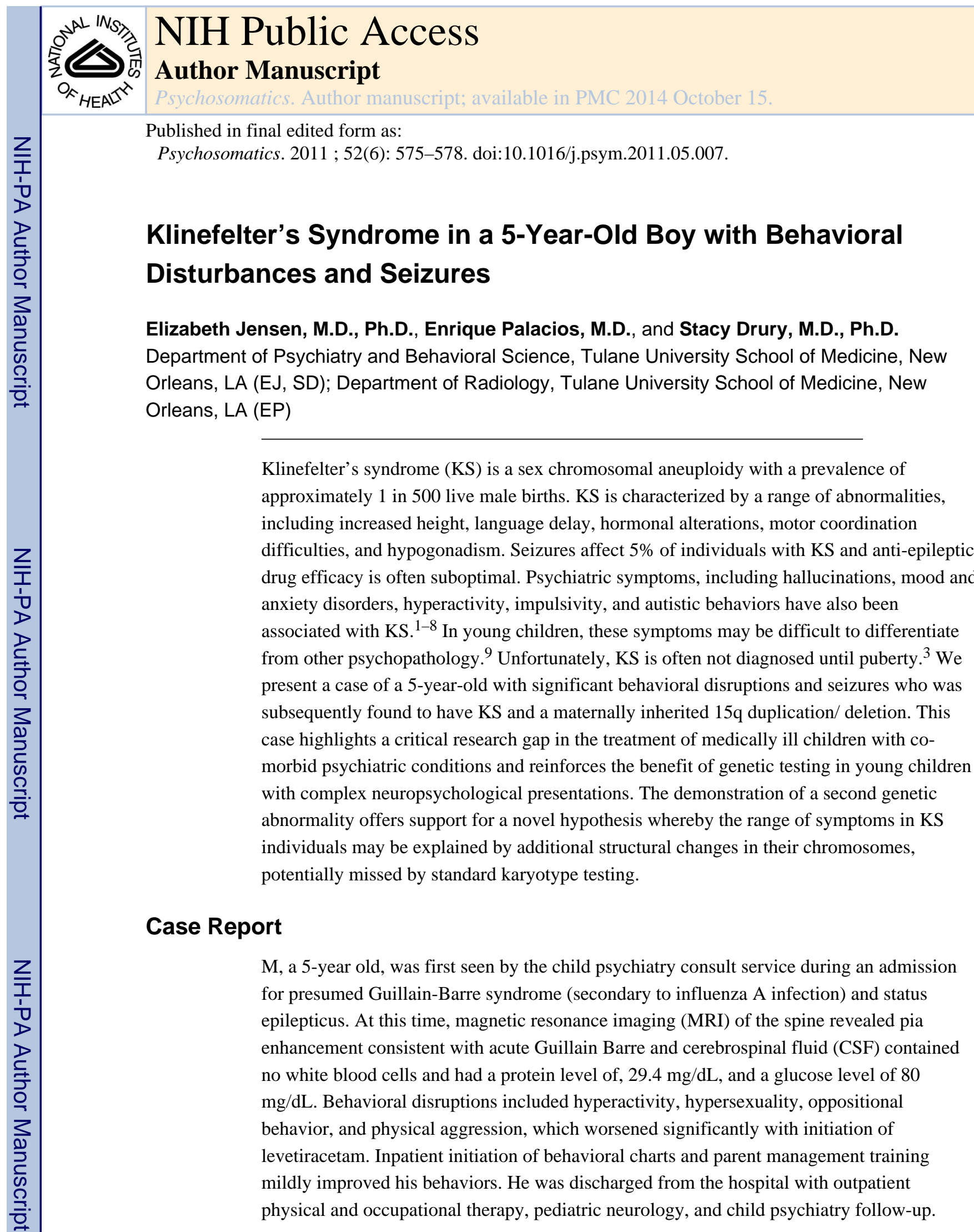

Klinefelter's syndrome (KS) is a sex chromosomal aneuploidy with a prevalence of approximately 1 in 500 live male births. KS is characterized by a range of abnormalities, including increased height, language delay, hormonal alterations, motor coordination difficulties, and hypogonadism. Seizures affect 5\% of individuals with KS and anti-epileptic drug efficacy is often suboptimal. Psychiatric symptoms, including hallucinations, mood and anxiety disorders, hyperactivity, impulsivity, and autistic behaviors have also been associated with KS. ${ }^{1-8}$ In young children, these symptoms may be difficult to differentiate from other psychopathology. ${ }^{9}$ Unfortunately, KS is often not diagnosed until puberty. ${ }^{3} \mathrm{We}$ present a case of a 5-year-old with significant behavioral disruptions and seizures who was subsequently found to have KS and a maternally inherited $15 \mathrm{q}$ duplication/ deletion. This case highlights a critical research gap in the treatment of medically ill children with comorbid psychiatric conditions and reinforces the benefit of genetic testing in young children with complex neuropsychological presentations. The demonstration of a second genetic abnormality offers support for a novel hypothesis whereby the range of symptoms in KS individuals may be explained by additional structural changes in their chromosomes, potentially missed by standard karyotype testing.

M, a 5-year old, was first seen by the child psychiatry consult service during an admission for presumed Guillain-Barre syndrome (secondary to influenza A infection) and status epilepticus. At this time, magnetic resonance imaging (MRI) of the spine revealed pia enhancement consistent with acute Guillain Barre and cerebrospinal fluid (CSF) contained no white blood cells and had a protein level of, $29.4 \mathrm{mg} / \mathrm{dL}$, and a glucose level of 80 $\mathrm{mg} / \mathrm{dL}$. Behavioral disruptions included hyperactivity, hypersexuality, oppositiona behavior, and physical aggression, which worsened significantly with initiation of levetiracetam. Inpatient initiation of behavioral charts and parent management training physical and occupational therapy, pediatric neurology, and child psychiatry follow-up.

Send correspondence and reprint requests to Elizabeth Jensen, M.D., Ph.D., Department of Psychiatry and Behavioral Science, Tulane University School of Medicine, 1440 Canal St., New Orleans, LA 70112-2715. ejensen@tulane.edu.

Disclosure: The authors disclosed no proprietary or commercial interest in any product mentioned or concept discussed in this article. 


\section{Developmental and Family History}

M's early development was unremarkable. There was no evidence of substance abuse, child abuse or neglect, or other traumatic experiences. Family history was significant for attention-deficit/hyperactivity disorder (ADHD) and a nonverbal learning disorder in his maternal half-sister. His mother had endometriosis, diabetes, hypothyroidism, depression, and post-traumatic stress disorder. His father had a history of a pulmonary embolism and depression.

\section{Previous Psychiatric and Behavioral Course}

Temper tantrums began at age 2 and his first intervention for behavioral disruption was at age 3. Prior to the age of 5 years, when evaluated by the consult service, $M$ had been treated with several stimulants, two different atypical antipsychotics, and an $\alpha$ agonist for presumed diagnoses of ADHD, oppositional defiant disorder, and mood disorder. Multiple medications for disruptive behavior were discontinued due to side effects (including the development of hypotension with guanfacine, oculomotor tics and obsessive compulsive symptoms with several stimulants), and a lack of efficacy (with topiramate, oxcarbazepine, and risperidone).

\section{Treatment Course}

Following his hospital admission, $\mathrm{M}$ was gradually tapered off levetiracetam and placed on valproic acid for seizure treatment while targeting disruptive behaviors. Discontinuation of the levetiracetam and implementation of individual therapy and behavioral management therapy resulted in moderate behavioral improvement and seizure control. However, the persistence of almost daily aggression and mood lability prompted augmentation with aripiprazole.

Several months later, $\mathrm{M}$ began kindergarten in a regular classroom setting. Within 3 weeks of starting school, $\mathrm{M}$ was hitting peers and teachers, throwing furniture, and faking seizures on a daily basis. An increase in aripiprazole from $2.5 \mathrm{mg}$ to $5 \mathrm{mg}$ resulted in gait instability and abnormal movements, which resolved when the dose was decreased to $2.5 \mathrm{mg}$. A retrial of stimulant medication resulted in better behavioral control, but also ataxia, dyscoordination, increased bilateral extremity weakness, and choreiform movements, so the stimulant was discontinued. However, neurologic symptoms persisted, resulting in hospital admission. An MRI and CSF analysis at this time were unremarkable. Electromyogram revealed mildly slowed left upper extremity sensory conduction velocities and low right sural amplitude. His neurologic symptoms resolved over the next week; however, thrombocytopenia necessitated discontinuation of valproic acid. The discontinuation of valproic acid resulted in a significant increase in behavioral disruption, mood instability, aggression, and oppositional behavior requiring physical and chemical restraints. He was subsequently transferred to an inpatient psychiatric hospital. Upon discharge from the psychiatric hospital, medications included lamotrigine, clorazepate, and aripiprazole. A well-defined behavior and de-escalation plan was implemented at home and school. Lamotrigine was titrated over the course of several months to $100 \mathrm{mg}$ twice a day, clorazepate to $3.75 \mathrm{mg}$ at night, and aripiprazole to $5 \mathrm{mg}$ twice a day. Parents began ParentChild Interaction Therapy (PCIT) ${ }^{10}$ targeting M's oppositional behavior. M continued 
Cognitive-Behavioral Therapy (CBT) focused on anxiety and mood symptoms ${ }^{11}$ along with outpatient physical and occupational therapy.

\section{Physical and Neurologic Examination and Laboratory Findings}

$M$ was greater than 95th percentile in height and weight and his genitourinary exam revealed descended but small testicles. Repeated neurologic exams demonstrated decreased fine and gross motor strength, persistent arreflexia and hypotonia, decreased coordination, and deficits in visual perception. On the Visual Motor Integration test, $\mathrm{M}$ scored in the 7th percentile, the 68th percentile for visual perception, and the 21st percentile for motor coordination. $\mathrm{M}$ had four witnessed seizures between the ages of 5 and 6 years. Repeated electroencephalograms (EEG) were negative. Repeated MRIs failed to identify causative structural abnormalities except for a benign unchanging $6 \mathrm{~mm}$ choroidal fissure cyst. Neuropsychiatric testing, which included the NEPSY-II, Reynolds Intellectual Assessment Scales, and gross and fine motor tasks revealed that $\mathrm{M}$ was capable of recognizing emotional facial expressions and was able to imitate hand positions in a sensorimotor task. While the majority of his scores were low-average or below, the validity was questionable given a high level of oppositional behavior during testing. At age 6, he was pre-reading and excelling in first grade spelling and mathematics with most of his classes in a regular setting. $\mathrm{He}$ exhibited no deficits in communication, no language delay, no repetitive movements, and no stereotypies. Thyroid studies, chemistries, porphobilinogen, transferrin, DNAse B antibody, ceruloplasmin, and copper levels were all within normal limits. Quantitative amino acid screening was negative. Karyotype analysis revealed $100 \%$ cells with XXY genotype, and comparative genomic hybridization $(\mathrm{CGH})$ demonstrated the presence of a $15 \mathrm{q} 13.3$ maternally-inherited duplication/deletion that was not present in his biological father and also not found in his half sister with a learning disorder.

\section{Discussion}

$\mathrm{KS}$ is a common chromosomal aneuploidy associated with complex medical and behavioral disruptions. Although fewer than $25 \%$ of patients are diagnosed prior to puberty, ${ }^{12}$ individuals with KS may exhibit behavioral problems earlier. M received multiple psychiatric diagnoses before age 5 and was highly treatment-resistant, yet genetic studies were not performed until he was seen by the child psychiatry consult service. Earlier genetic diagnosis of KS may have prompted more extensive neuropsychological testing and subsequent earlier intervention for his deficits in gross and fine motor skills, which often contribute to poor academic functioning and disruptive behavior. The presence of a second genetic structural anomaly, the $15 q 13$ duplication deletion ${ }^{13}$ independently associated with neurocognitive deficits, seizures, autism, and schizophrenia, raises the interesting possibility that children with KS may have additional genomic rearrangements that contribute to the heterogeneity of symptoms.

Research in the use of medications in KS is limited. ${ }^{3,4} \mathrm{M}$ exhibited significant worsening of disruptive behavior on levetiracetam, developed thrombocytopenia on valproic acid, and had a transient rash on lamotrigine. Clorazepate was an effective adjuvant. ${ }^{14} \mathrm{M}$ also experienced side-effects to agents that impact the mesolimbic dopamine levels. Increase in aripiprazole 
dose, a dopamine partial agonist-antagonist, resulted in onset of choreoathetoid movements and ataxia. Both methylphenidate and dexamphetamine stimulant medications were discontinued due to neurologic side-effects. Further evidence supporting disturbances of the mesolimbic dopamine pathway with KS include the prevalence of tremor in $\mathrm{KS},{ }^{15}$ and the finding that testosterone administration reduces the tremor. ${ }^{16}$ Collectively, these findings suggest the possibility of alterations in the mesolimbic dopamine motor pathway in KS.

The cross-domain symptoms of KS, including motor, neurologic, and behavioral symptoms, present a challenge for effective interventions. M's treatment required the implementation of an evidence-based treatment for disruptive behavior in preschool children (PCIT), individual CBT, physical and occupational therapy, and a carefully constructed educational and behavioral plan. While few evidence-based treatments exist for preschool children, PCIT has a significant evidence base for oppositional behavior in medically ill children, ${ }^{17}$ and there is an increasing evidence base for the use of CBT in preschool children. ${ }^{11}$

This case report identifies a critical gap in pediatric psychosomatic medicine. There are no randomized controlled psychopharmacologic trials in medically ill children. This gap is compounded in preschool children, where psychotropic medications are increasingly prescribed, the evidence base for their use is scarce, and the incidence of medication side effects is elevated. ${ }^{18-20}$ Psychopharmacologic interventions in preschool children, whether medically ill or not, should be used only within the context of a well-defined comprehensive multi-modal treatment plan. ${ }^{18}$ This case demonstrates the utility of genetic studies in the evaluation of young children with both neurologic and behavioral problems, highlights a critical research gap in pediatric psychosomatic medicine, and outlines a comprehensive evidence-based approach to the treatment of preschool children with comorbid medical and psychiatric conditions.

\section{Acknowledgments}

Funding: NARSAD Young Investigator Award (SD), Tulane University CTREC (SD).

\section{References}

1. van Rijn S, Aleman A, Swaab H, et al. Klinefelter's syndrome (karyotype 47, XXY) and schizophrenia-spectrum pathology. Br J Psychiatry. 2006; 189:459-460. [PubMed: 17077438]

2. Bojesen A, Juul S, Birkebaek NH, et al. Morbidity in Klinefelter's syndrome: a Danish register study based on hospital discharge diagnoses. J Clin Endocrinol Metab. 2006; 91:1254-1260. [PubMed: 16394093]

3. Tatum WO, Passaro EA, Elia M, et al. Seizures in Klinefelter's syndrome. Pediatr Neurol. 1998; 19:275-278. [PubMed: 9830997]

4. Grosso S, Farnetani MA, Di Bartolo RM, et al. Electroencephalographic and epileptic patterns in X chromosome anomalies. J Clin Neurophysiol. 2004; 21:249-253. [PubMed: 15509914]

5. Elia M, Musumeci SA, Ferri R, et al. Seizures in Klinefelter's syndrome: a clinical and EEG study of five patients. Ital J Neurol Sci. 1995; 16:231-238. [PubMed: 7591675]

6. Yang CY, Lin KC, Chou CW, et al. Klinefelter's syndrome with seizure, pseudohypoparathyroidism type Ib and multiple endocrine dysfunctions. J Chin Med Assoc. 2005; 68:585-590. [PubMed: 16379343]

7. Merhar SL, Manning-Courtney P. Two boys with 47, XXY and autism. J Autism Dev Disord. 2007; 37:840-846. [PubMed: 16927010] 
8. Bruining H, Swaab H, Kas M, et al. Psychiatric characteristics in a self-selected sample of boys with Klinefelter syndrome. Pediatrics. 2009; 123:e865-870. [PubMed: 19364768]

9. DeMaso DR, Martini DR, Cahen LA, et al. Work Group on Quality, Issues. Practice parameter for the psychiatric assessment and management of physically ill children and adolescents. J Am Acad Child Adolesc Psychiatry. 2009; 48:213-233. [PubMed: 20040826]

10. Schuhmann EM, Foote RC, Eyberg SM, et al. Efficacy of parent-child interaction therapy: interim report of a randomized trial with short-term maintenance. J Clin Child Psychol. 1998; 27:34-45. [PubMed: 9561935]

11. Scheeringa MS, Salloum A, Arnberger RA, et al. Feasibility and Effectiveness of CognitiveBehavioral Therapy for Posttraumatic Stress Disorder in Preschool Children: Two Case Reports. J Trauma Stress. 2007; 20:631-636. [PubMed: 17721975]

12. Geschwind DH, Boone KB, Miller BL, et al. Neurobehavioral phenotype of Klinefelter syndrome. Ment Retard Dev D R. 2000; 6:107-116.

13. Michelson M, Eden A, Vinkler C, et al. Familial partial trisomy 15q11-13 presenting as intractable epilepsy in the child and schizophrenia in the mother. Eur J Paediatr Neurol. 2011; 15:230-233. [PubMed: 21145272]

14. Riss J, Cloyd J, Gates J, et al. Benzodiazepines in epilepsy: pharmacology and pharmacokinetics. Acta Neurol Scand. 2008; 118:69-86. [PubMed: 18384456]

15. Harlow TL, Gonzalez-Alegre P. High prevalence of reported tremor in Klinefelter syndrome. Parkinsonism Related Dis. 2009; 15:393-395.

16. Kinoshita H, Ohkubo T, Kaneko M, et al. Tremor in Klinefelter's syndrome improved by testosterone administration. J Neurol. 2009; 256:1924-1925. [PubMed: 19597693]

17. Bagner DM, Fernandez MA, Eyberg SM. Parent-child interaction therapy and chronic illness: a case study. J Clin Psychol Med Settings. 2004; 11:1-6.

18. Gleason MM, Egger HL, Emslie GJ, et al. Psychopharmacological treatment for very young children: contexts and guidelines. J Am Acad Child Adolesc Psychiatry. 2007; 46:1532-1572. [PubMed: 18030077]

19. Olfson M, Crystal S, Huang C, et al. Trends in antipsychotic drug use by very young, privately insured children. J Am Acad Child Adolesc Psychiatry. 2010; 49:13-23. [PubMed: 20215922]

20. Greenhill L, Kollins S, Abikoff H, et al. Efficacy and safety of immediate-release methylphenidate treatment for preschoolers with ADHD. J Am Acad Child Adolesc Psychiatry. 2006; 45:1284 1293. [PubMed: 17023867] 